\title{
Multidetector CT evaluation in Gastro-Intestinal Bleeding
}

\author{
Dr. Anisha Gehani ${ }^{1}$, Dr. Viral Patel ${ }^{2}$, Dr. Jayesh Bhatt ${ }^{3}$ \\ ${ }^{1}$ Radiology Resident, ${ }^{2}$ Associate Professor, ${ }^{3}$ Professor and Head-Department of Radiodiagnosis, Shree Krishna \\ Hospital, Pramukhswami Medical College, Karamsad, Gokalangar, Anand, Gujarat, India -388325
}

\begin{abstract}
Gastrointestinal bleeding is a common and complex condition which requires a multispecialty approach for diagnosis, evaluation and management. The length of gastrointestinal tract as well as the variety of processes that can involve the gastrointestinal tract further complicate the evaluation. MDCT is a recently developed diagnostic tool for the evaluation of bowel diseases causing gastrointestinal bleeding. It not only helps in defining the extent of gastrointestinal disease, it also helps in evaluation of extra intestinal manifestations of the disease. Study was conducted by performing CT scan of abdomen and pelvis on a 64 Slice Optima CT 660 (Wipro GE Healthcare Pvt. Ltd.). The CT examination revealed pathological findings in 29 (80.55\%) out of the 36 patients which were bowel wall thickening in 19 (52.8\%), mass lesion in 14 (38.9\%), polyp in $2(5.5 \%)$, diverticulitis in 1 (2.7\%), colitis in 1 (2.7\%), varices in 1 (2.7\%), hemorrhoids in 1 (2.7\%) and vascular aneurysm in 1 (2.7\%) patient with gastrointestinal bleeding. The maximum number of patients i.e. $9(25 \%)$ were in the age group of 71-80 years. There was a female predominance with 23 (63.88\%) females and $13(36.11 \%)$ males.
\end{abstract}

Keywords: bowel wall thickening, gastrointestinal bleeding, gastrointestinal polyps, MDCT abdomen and pelvis, mass lesion,.

\section{Introduction}

Gastrointestinal bleeding is a common cause of hospitalization, morbidity, and mortality. The evaluation of gastrointestinal bleeding requires a multispecialty approach involving physicians, surgeons, and radiologists. The length of the gastrointestinal tract, the great number of pathologic processes causing gastrointestinal bleeding, as well as intermittent nature of gastrointestinal bleeding make it complex process for evaluation.

Mutlidetector CT is a recently developed diagnostic tool for the evaluation of bowel diseases causing gastrointestinal bleeding. ${ }^{(1,2)}$ This technique uses multi-detector CT combined with luminal distention of the bowel by using enteric contrast material, which may be of two types; positive or neutral, depending on the suspected underlying aetiology. ${ }^{(3)}$ Oral contrast would help to optimize visualization of intraluminal aetiologies. Image acquisition after intravenous contrast material administration is timed to optimize any abnormal bowel wall enhancement. Multidetector CT systems (like 64 and 128 slice systems) permit an increase in both spatial and temporal resolution, rapid acquisition and a large volume to be scanned. Furthermore, triple phase scanning technique aids in the diagnosis of extra-intestinal manifestations. ${ }^{(4,5)}$ The advantages of this technology seem well suited for the investigation of gastrointestinal bleeding due to its speed, resolution, multi-planar techniques, and angiographic capabilities.

Thus, an adequately performed contrast MDCT examination is a promising first-line modality for the time-efficient, sensitive, and accurate diagnosis of the primary disease and determining the presence, location and severity of complications as a result of the disease. This study has had had profound impact on the subsequent treatment line of patients who present with gastrointestinal bleeding in a tertiary health care centre in rural India.

\section{Aims And Objectives}

To study the spectrum of imaging observations depicted at CT performed with a Multi Detector CT system and by using enteric contrast material and a three-phase acquisition in patients with gastrointestinal bleeding in patients of gastro-intestinal bleeding coming to the radiology department of Shree Krishna Hospital, Anand.

\section{Materials And Methods}

36 symptomatic patients presenting with gastro-intestinal bleeding (based on clinical history / endoscopy reports / stool test) referred to the Radiology department of Shree Krishna Hospital were subjected to CT scan of abdomen and pelvis. The study included patients of both sex, irrespective of their religion, age or socioeconomic status.Study was performed with CT scan machine (64 Slice Optima CT 660 (Wipro GE Healthcare Pvt. Ltd.) 


\subsection{Exclusion Criteria}

- Pregnant women.

- Contra indications to contrast- such as suspected or known case of anaphylaxis, elevated serum creatinine / GFR, multiple myeloma, sickle cell disease.

- $\quad$ Critically ill patients.

\subsection{Technique of examination}

The procedure was briefly explained to the patient including the risks of contrast examination. Clinical history of each patient was recorded and informed consent was taken. $750 \mathrm{ml}$ of Oral contrast (positive trazograff / neutral - mannitol) was given 60-90 minutes prior to the CT scan. $250 \mathrm{ml}$ of same contrast was administered by per-rectal enema for opacification of large bowel loops. A topogram of the abdomen and pelvis was obtained and series were planned according to contrast enhanced CT scan protocol for abdomen and pelvis.

With patient in supine position, non-contrast $7.5 \mathrm{~mm}$ thick contiguous helical MDCT sections of abdomen and pelvis were taken from the domes of diaphragm to ischial tuberosity after opacification of bowel loops.After intravenous administration of $100 \mathrm{ml}$ of non-ionic iodinated contrast medium (300 mg iodine $/ \mathrm{ml}-$ Iohexol), $7.5 \mathrm{~mm}$ contiguous helical MDCT sections were obtained in arterial phase (at 25-35 seconds), portal phase (at 40-55 seconds) and venous phase (at 70 seconds). Few $7.5 \mathrm{~mm}$ thick delayed helical MDCT sections will also be taken through the region of interest.Few coronal \& sagittal reconstructions were also done wherever required. As a precautionary measure, resuscitation apparatus and emergency drugs were kept ready.

\section{Method of Data Analysis}

Collected data was presented in the form of tables and diagrams. Frequency and percentages were calculated wherever applicabe.

\section{Results}

In my study, 36 patients with clinical history of gastrointestinal bleeding were studied by contrast enhanced CT scan of abdomen and pelvis. The age range of patients was from 21 years to 85 years and the maximum number of patients i.e. $9(25 \%)$ were in the age group of 71-80 years (table 1). There was a female predominance with $23(63.88 \%)$ females and $13(36.11 \%)$ males.

TABLE 1- Age Distribution

\begin{tabular}{|l|l|l|}
\hline Age & Number of Patients & Percentage \\
\hline $21-30$ YEARS & 2 & 5.5 \\
\hline $31-40$ YEARS & 4 & 11.1 \\
\hline $41-50$ YEARS & 7 & 19.4 \\
\hline $51-60$ YEARS & 8 & 22.2 \\
\hline $61-70$ YEARS & 5 & 13.8 \\
\hline $71-80$ YEARS & 9 & 25 \\
\hline$>80$ YEARS & 1 & 2.7 \\
\hline
\end{tabular}

The study revealed pathological findings in 29 patients of which bowel wall thickened and mass lesion were the most frequent findings followed by rest of the findings as tabulated in table 2.

TABLE 2- Spectrum of CT findings

\begin{tabular}{|l|l|l|}
\hline Finding on CT scan & Number of patients & Percent \\
\hline MASS LESION & 14 & 38.9 \\
\hline BOWEL WALL THICKENING & 19 & 52.8 \\
\hline DIVERTICULITIS & 1 & 2.7 \\
\hline POLYP & 2 & 5.5 \\
\hline COLITIS & 1 & 2.7 \\
\hline VASCULAR ANEURYSM & 1 & 2.7 \\
\hline VARICES & 1 & 2.7 \\
\hline HEMORRHOIDS & 1 & 2.7 \\
\hline ANGIODYSPLASIA & 0 & - \\
\hline NORMAL & 7 & 19.4 \\
\hline
\end{tabular}

Patients presented with different types of gastrointestinal bleeding with the maximum number i.e., 26 (72\%) of the total presenting with malena (table 3 ). 
TABLE 3- Type of Bleeding

\begin{tabular}{|l|l|l|}
\hline & Frequency & Percent \\
\hline HEMATEMESIS & 5 & 13.9 \\
\hline MALENA & 24 & 66.6 \\
\hline HEMATOCHEZIA & 5 & 13.9 \\
\hline HEMATEMESIS AND MALENA & 2 & 5.6 \\
\hline
\end{tabular}

The present study was able to accurately detect the site of pathology as correlated with the type of bleeding which is as tabulated below.

TABLE 4- Type of bleeding correlation with site of pathology

\begin{tabular}{|l|l|l|}
\hline Type of Bleeding & Site of Pathology & Percentage \\
\hline HEMATEMESIS & Above ligament of Treitz & $80 \%$ \\
\hline MALENA & Below ligament of Treitz & $78.9 \%$ \\
\hline HEMATOCHEZIA & Distal colon and Ano-rectum & $80 \%$ \\
\hline
\end{tabular}

For staging and further management decision, CT also revealed extra-intestinal manifestations findings such as surrounding fat infiltration in 16 (44.4\%), adjacent organ involvement in 9 (25\%), adjacent vascular involvement in $4(11.1 \%)$, lymphadenopathy in $19(52.7 \%)$, hepatic metastases in $10(27.7 \%)$, musculoskeletal metastases in $2(5.5 \%)$ and lung metastases in $3(8.3 \%)$ patients with gastro-intestinal bleeding.

Table 5- Spectrum of Extra Intestinal Manifestations

\begin{tabular}{|l|l|l|}
\hline Extra intestinal Manifestations & Frequency & Percent \\
\hline FAT INFILTRATION & 16 & 44.4 \\
\hline ADJACENT ORGAN INVOLVEMENT & 9 & 25 \\
\hline ADJACENT VASCULAR INVOLVEMENT & 4 & 11.1 \\
\hline LYMPHADENOPATHY & 19 & 52.7 \\
\hline HEPATIC METASTASES & 10 & 27.7 \\
\hline MUSCULOSKELETAL METASTASES & 2 & 5.5 \\
\hline LUNG METASTASES & 3 & 8.3 \\
\hline
\end{tabular}

\section{Discussion}

The common findings as depicted in the CT in descending order of frequency areare as belows;

1. Bowel wall thickening

2. Mass lesion

3. Polyp

4. Diverticulitis, colitis, varices, hemorrhoids and vascular aneurysms.

\section{(i) Bowel Wall Thickening}

$19(52.8 \%)$ patients had shown evidence of bowel wall thickening on CT scan- which was also more frequently found in the large bowel. 3 patients had shown heterogeneously enhancing malignant wall thickening involving pylorus of stomach (Fig.1) and 2 patients had asymmetric wall thickening of the gastro-esophageal junction and greater curvature of stomach. In 1 of them, CT was also able to demonstrate gastric outlet obstruction as a complication due to the diffuse wall thickening and pre-pyloric large lymph nodes.

A confirmed diagnosis of malignant wall thickening could be given due to the presence of thickening measuring greater than $1 \mathrm{~cm}$, which was also confirmed by associated findings such as large necrotic and heterogeneously enhancing lymph nodes and fat stranding which were in in concordance with a study ofInsko et al $2003^{(6)}$ which also shows the upper cut off for malignant wall thickening to be $1 \mathrm{~cm}$.

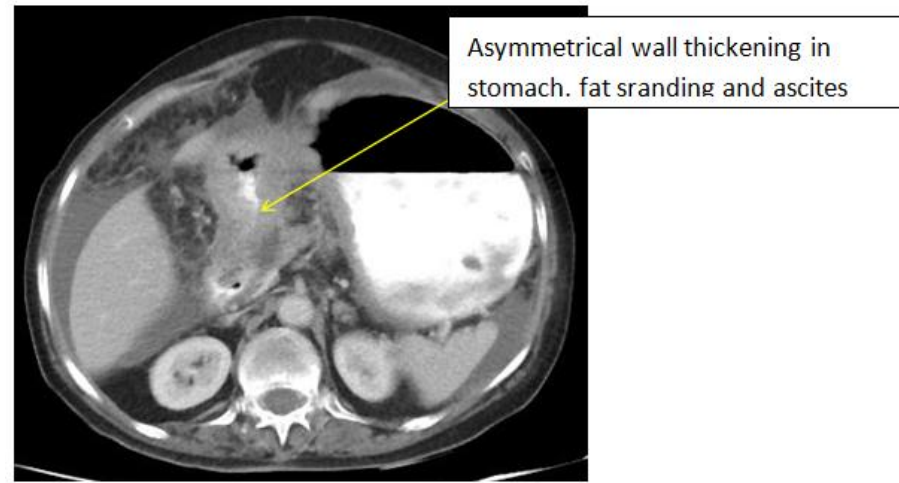

Figure 1 - a case of wall thickening of stomach causing hematemesis and malena. 
1 patient had shown diffuse extra-luminal wall thickening involving the $2^{\text {nd }}$ and $3^{\text {rd }}$ part of duodenum which was associated with large retro-peritoneal lymphadenopathies together which were encasing the superior mesenteric axis favoring small bowel lymphoma (Fig.-2). Serour F. et al (1992) ${ }^{(7)}$ in his study of 61 patients with small bowel tumours had concluded lymphoma as most common primary neoplasm of small bowel in 38.6 $\%$. The most favoured site of small bowel lymphoma was distal ileum, however, in mystudy, occurrence of lymphoma was in the duodenum ${ }^{(8)}$.
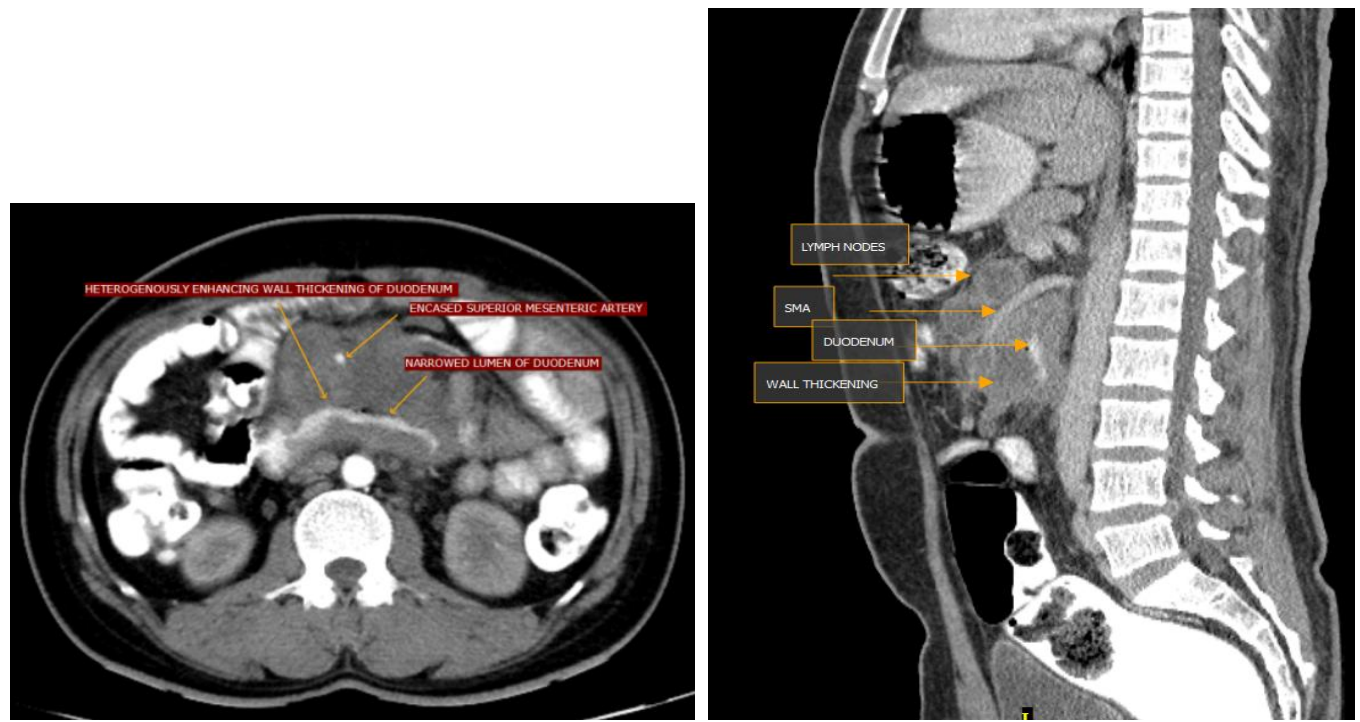

Figure 2 - A case of lymphoma of small bowel causing malena

2 patients had shown circumferential wall thickening of an infective etiology involving the terminal ileum, ileo-cecal junction and ascending colon in conordance with study done by Theoni et al (2006) ${ }^{(9)}$ depicting continuous and symmetrical wall thickening in colitis. 1 patient had shown homogenously enhancing diffuse symmetric thickening of ascending colon due to inflammatory etiology (he was a known case of pancreatitis).

10 patients had asymmetrical, irregular, heterogonous wall thickening involving large bowel. Of which, 1 patient had asymmetrical short segmental heterogeneously enhancing malignant wall thickening in the mid portion of the ascending colon (Fig. 3a-d), 2 patients had shown asymmetrical, irregular, heterogeneously enhancing, malignant wall thickening in the distal sigmoid colon and 7 patients had shown evidence of malignant wall thickening involving the rectum and anal canal. Of these, 1 patient had a positive history of familial adenomatous polyposis while in another patient, CT could rightly identify a rectovaginal fistula that had occurred as a complication. Of these 10 patients, 7 of them had shown involvement of the surrounding fat favoring advanced disease. To substantiate this, a study done by $\mathrm{Ng}$ et al $(2002)^{(10)}$ had shown the appearance of abnormal pericolic fat on CT to have a sensitivity of $79 \%$ and positive predictive value of $91 \%$ in identifying tumour infiltration beyond the muscle.

In my study, it was observed that females were more commonly affected than males by colorectal lesions with most commonly affected age group between 61-70 years. Although the age group affected was in concordance with a study done by Rajesh et al (2010) ${ }^{(11)}$, the pattern of gender distribution differed from his study, as it was found that males were more commonly affected $(53.71 \%)$ in his study. Rectum was the commonest site for malignant lesions in my study $(47.3 \%)$. This finding was in agreement with the study done by Rajesh et al $(2010){ }^{(11)}$.In their study there was involvement of the rectum in $53.71 \%$ of the cases.
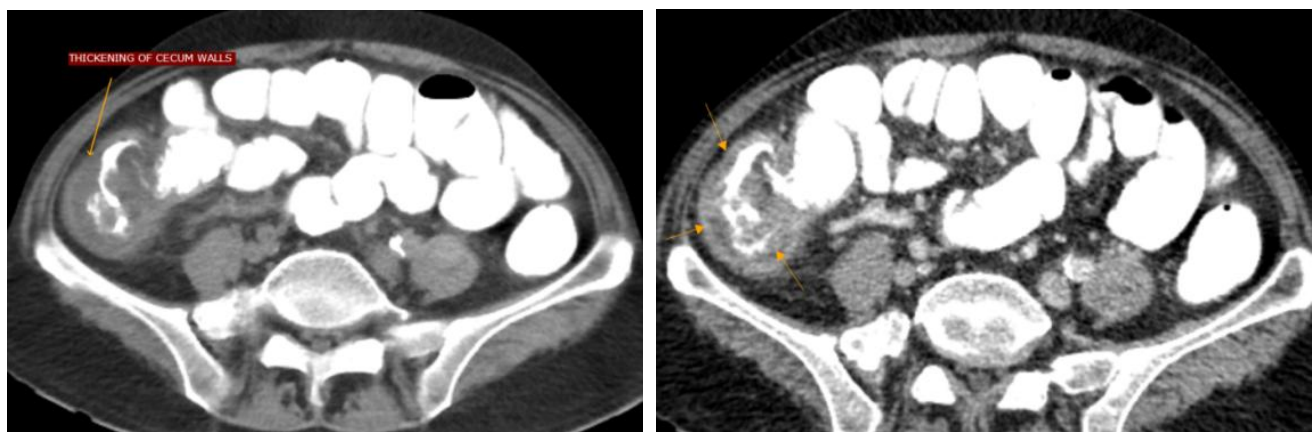

Fig 3(a,b) non-contrast and contrast enhanced venous phase axial images 


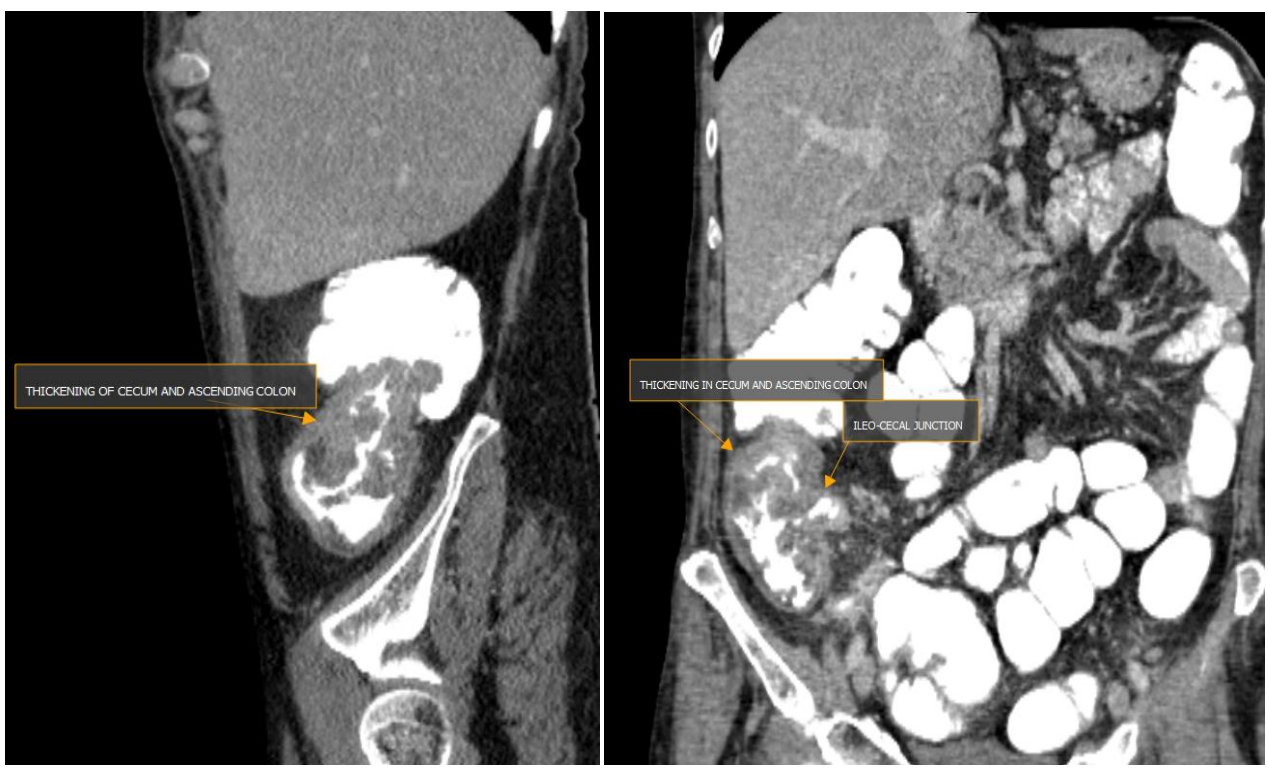

Fig 3(c,d) contrast enhanced venous phase sagital and coronal images

Figure 3 (a-d)- A case of large bowel wall thickening causing malena

\section{(ii) Mass Lesion}

14 patients $(38.9 \%)$ had mass lesions of the gastro-intestinal tract, of which $37.5 \%$ were in the age group of 41-50 years. Of the 14 patients with mass lesions in the gastro-intestinal tract, $50 \%$ were in the pylorus of the stomach (Fig. 4) while rectum was second most commonly involved site (35.7\%).

$64.2 \%$ patients with mass lesion presented with malena and had a comparatively earlier age of presentation at 41-50 years as compared with malignant wall thickening who presented at 61-70 years which was largely due to luminal narrowing effect and early obstructive complications as was seen in approximately 4 of these patients $(28.5 \%)$.

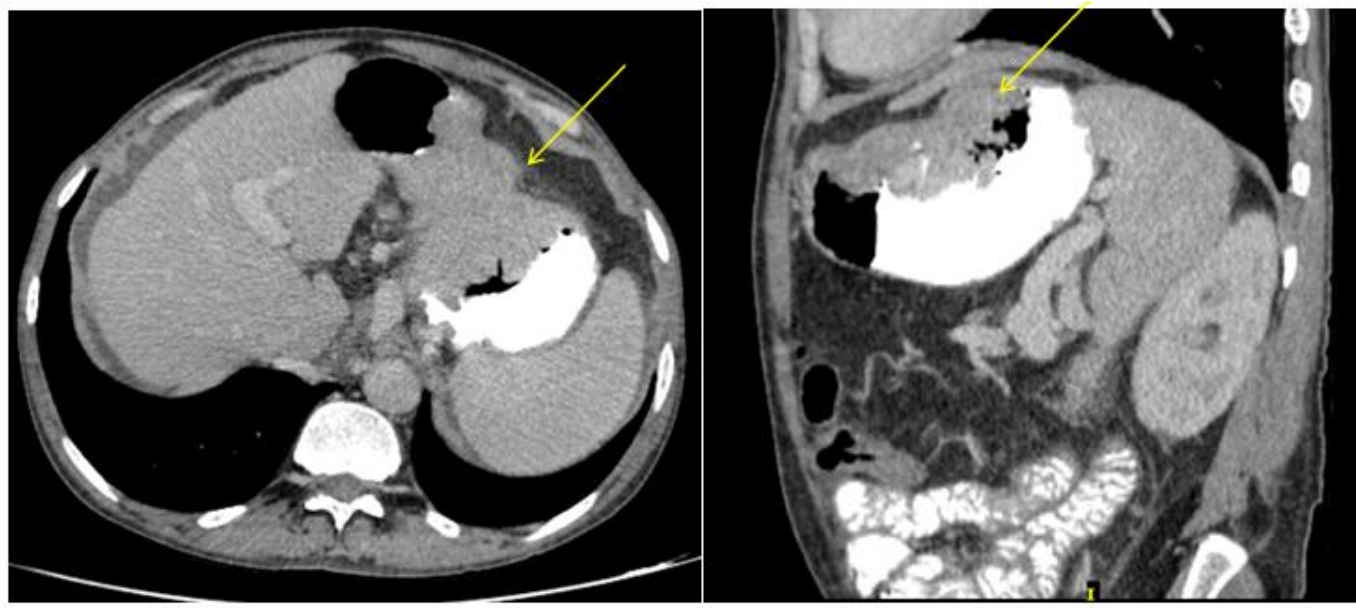

Figure 4 - a case of mass lesion of stomach causing hematemesis

\section{(iii) Polyp}

2 patients $(5.5 \%)$ had polyps in the gastrointestinal tract. Both the patients had presented with melena and both had multiple polyps at the time of presentation as they had undergone flexible colonoscopy prior to the CT scan. 1 patient had multiple varying sized polyps in the transverse, descending colon, sigmoid colon and rectum (Fig. 5). The biggest polyp was at the recto-sigmoid junction and measures $4 \mathrm{~cm}$. On CT scan, the polyp had a heterogeneous enhancement pattern and had an irregular margin which favored malignancy. Histopathologic correlation from the biopsy of the recto-sigmoid junction polyp had shown tubule-villous adenoma with high grade dysplasia. The patient was 39 years old. 


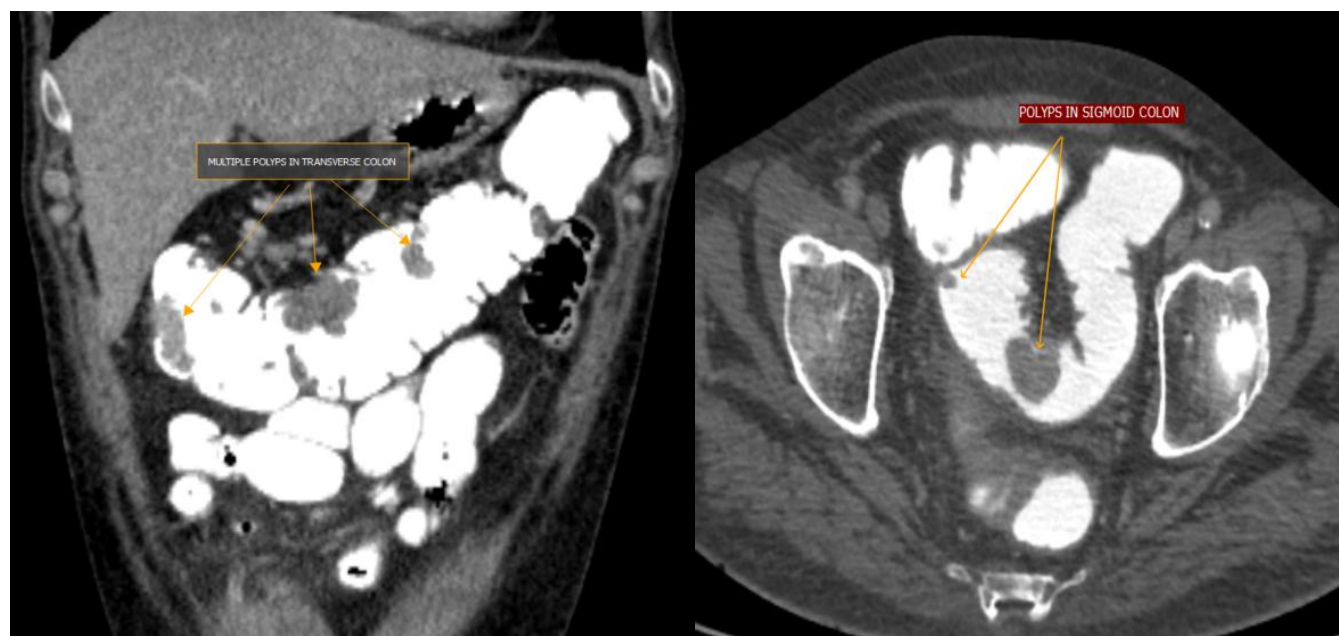

Figure 5- a case of multiple colonic polyposis causing malena

1 patient had multiple small polyps in the entire colon and two large polyps (measuring $>2.6 \mathrm{~cm}$ ) near the hepatic flexure and in the cecum. On CT scan, the multiple small polyps scattered diffusely throughout the bowel had shown homogenous contrast enhancement, while the bigger polyps in the cecum and hepatic flexure of colon had shown irregular margins, intraluminal as well as extra-luminal extension and heterogeneous contrast enhancement favoring malignancy. Histopathological correlation from colonoscopy guided biopsy of lesion in cecum and hepatic flexure had shown tubular and tubule-villous adenomas with dysplastic changes.

Thus, my study shows a positive correlation between site, size as well as histological type of colorectal polyps with presence of dysplasia and subsequent carcinomas. The occurence of polyps more commonly in the left side of the colon and rectum is in concordance with the sduies done by Sandra Beatriz Marion Valarini et al $(2011)^{(12)}$ and Khatibzadeh N. et al (2005) ${ }^{(13)}$. In their study as well, polyps in ascending and transverse colons correlated with dysplasia and those in the left colon and rectum were associated with adenocarcinoma. Lesions greater than $1 \mathrm{~cm}$ related to dysplasia and neoplasm. Adenomas were considered pre-malignant.

\section{(iv) Colitis}

1(2.7\%) patient had mild wall thickening associated with loss of haustral pattern of sigmoid colon, descending colon and left half of transverse colon and stricture formation at the mid transverse colon level.There were also bulky necrotic peripherally rim enhancing lymph nodes in the abdomen and thus a diagnosis of tubercular etiology was given for the same (Fig. 6).The patient was 72 years old, had a past history of pulmonary tuberculosis and had presented with low grade fever, abdominal pain and malena. Histopathological correlation from biopsy had shown chronic granulomatous inflammation suggestive of mycobacteriosis. In a study involving 40 patients with gastro-intestinal bleeding, tuberculosis was responsible for bleeding in $10 \%$ patients ${ }^{(14)}$.

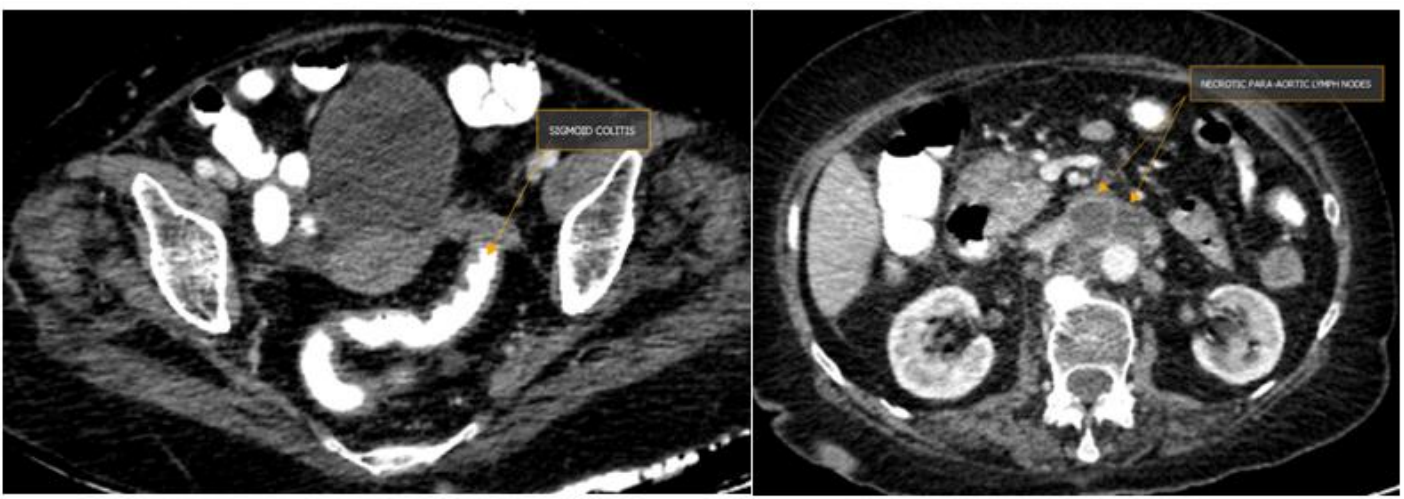

Fig.6: a,b- axial contrast enhanced images 


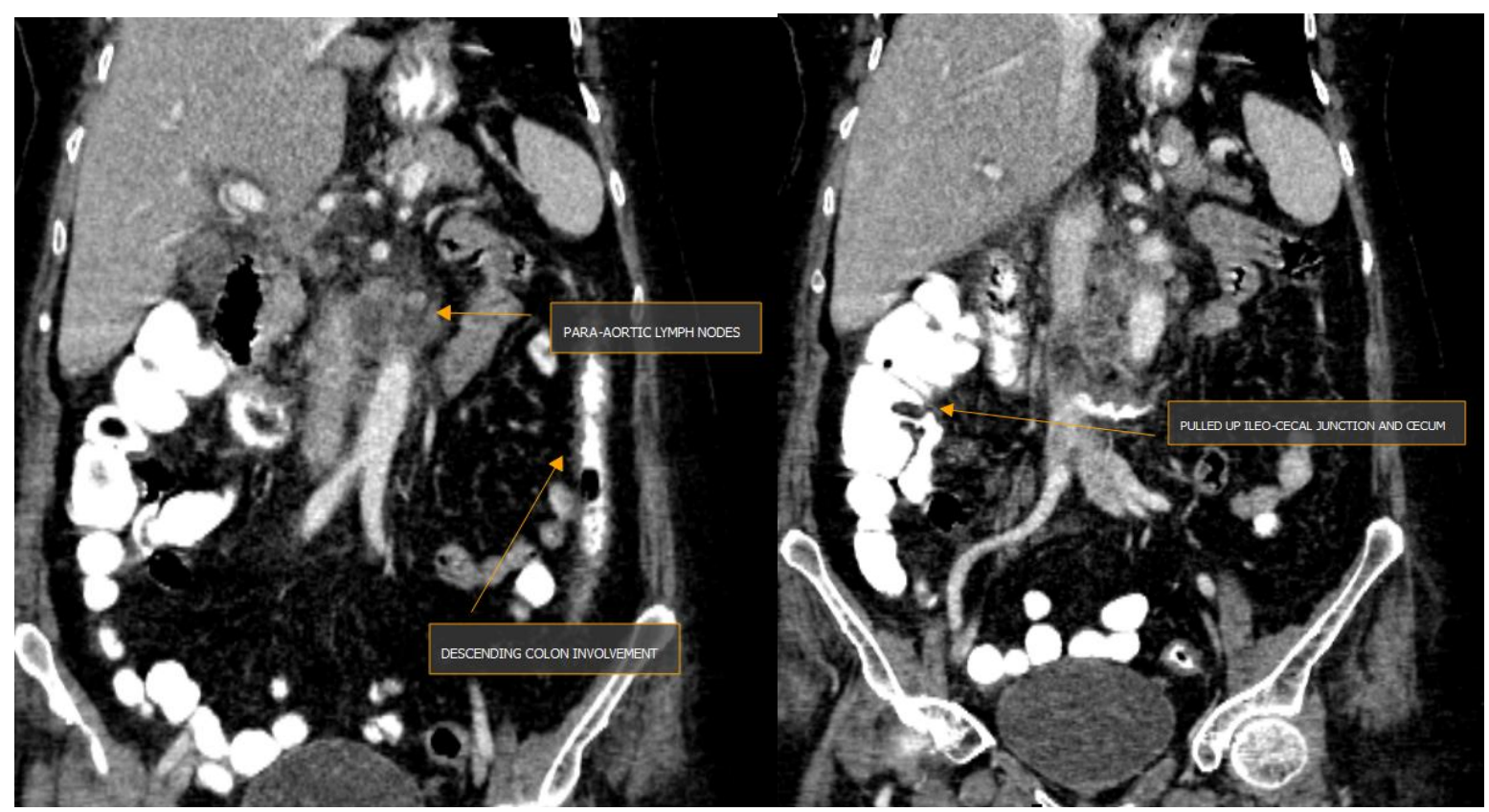

Fig.6- c,d- coronal contrast enhanced images

Figure 6(a-d) -A case of descending colon and sigmoid colitis causing malena

\section{(v) Hemorrhoids}

1(2.7\%) patient had shown heterogeneously enhancing thickening along the anal canal suggestive of hemorrhoids on CT scan. Clinically, the patient had a small chronic ulcer in the anal region with actively bleeding hemorrhoids on touch. In Asia, hemorrhoids are a common cause of lower gastro-intestinal bleeding followed by anal fissures and colorectal neoplasms. ${ }^{(19)}$

\section{(vi) Diverticulitis}

$1(2.7 \%)$ patient had shown multiple, small, diverticuli in the sigmoid colon and descending colon with few of the diverticuli in the descending colon showing wall edema, heterogeneous enhancement and peri-colic fat stranding favoring diverticulitis on CT scan (Fig. 7).

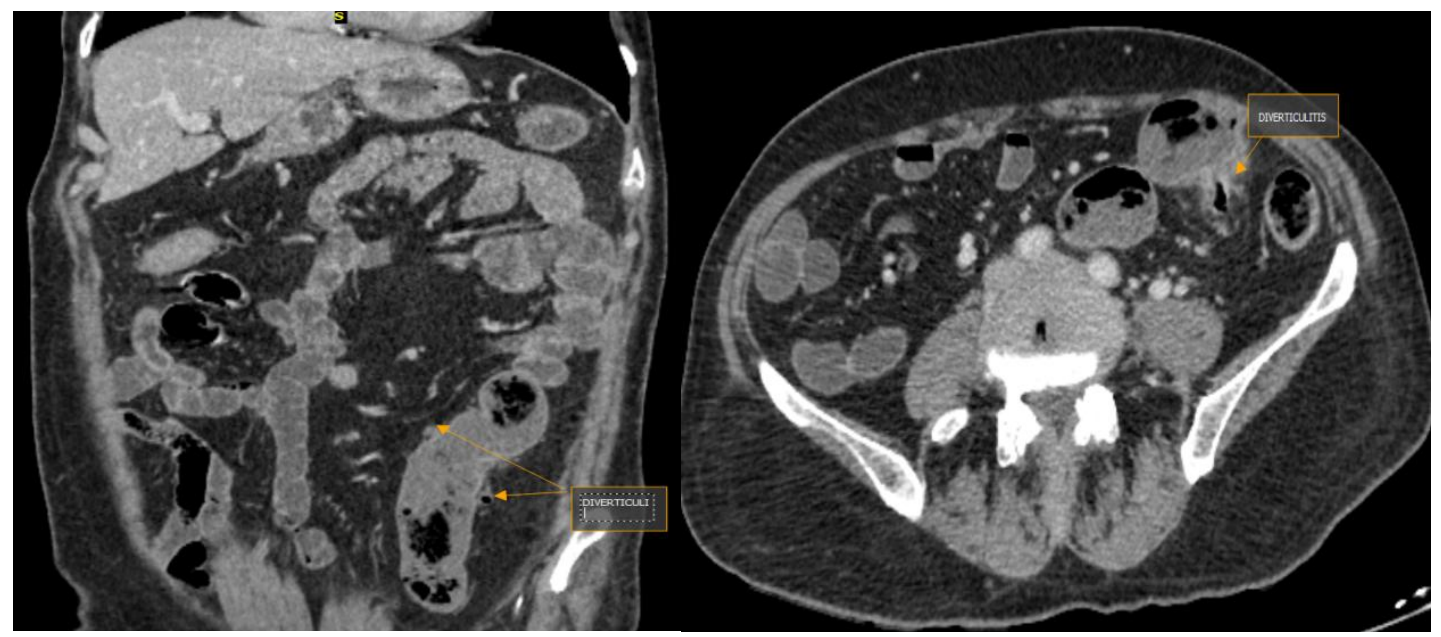

Figure 7- A case of colonic divertucilitis

\section{(vii) Vascular Causes}

$1(2.7 \%)$ patient had a saccular aneurysm of distal portion of gastro-duodenal artery (Fig. 8). The patient had acute onset hematemesis. She had pancreatitis and a pancreatic pseudocyst for which she underwent cystogastrostomy. The aneurysm was found at the operative site along the posterior wall of distal body of stomach. In my study, there were no cases of angiodysplasia or Dilefouy's lesion. This was discordant with a systematic review by Liao et al. (2010) ${ }^{(18)}$ that included 227 studies whereby angiodysplasia was the most common underlying lesion $(50 \%)$ in patients presenting with gastrointestinal bleeding. 


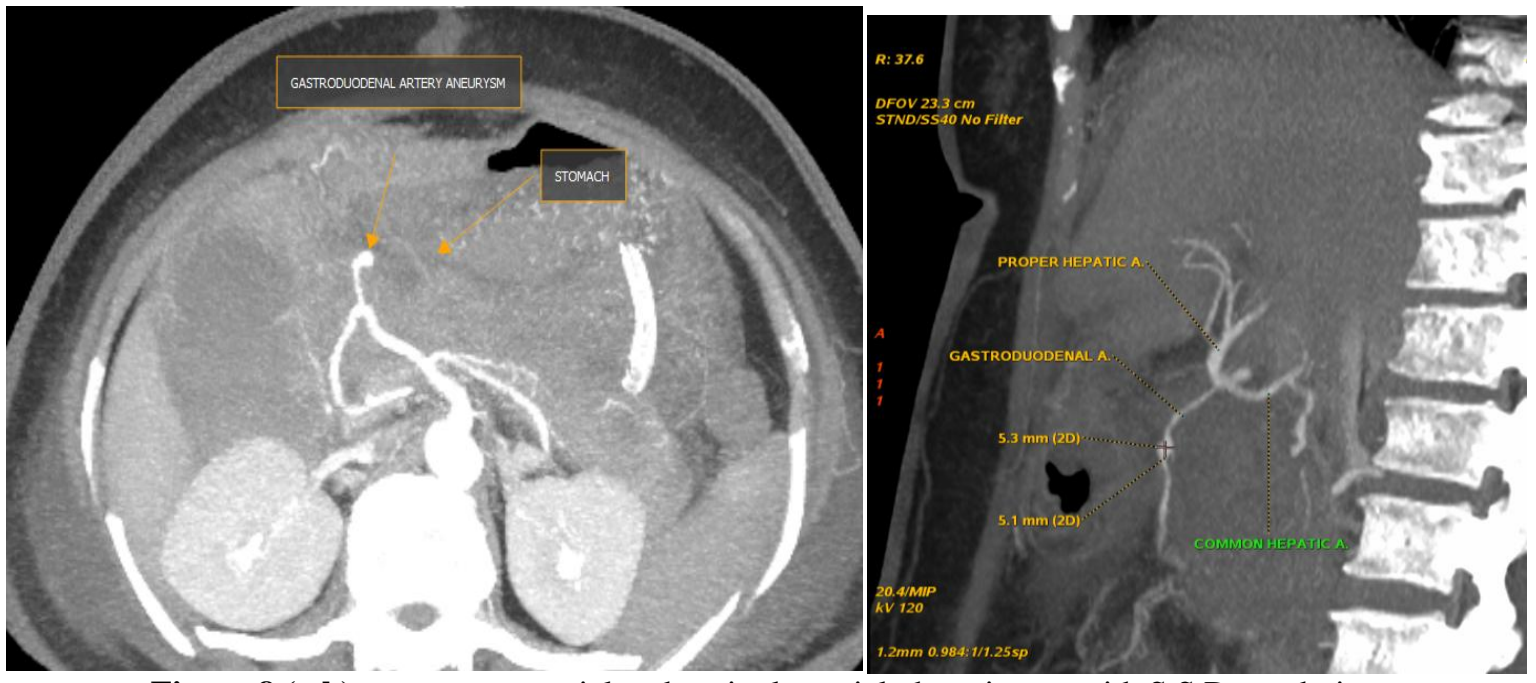

Figure $8(\mathbf{a}, \mathbf{b})$ post contrast axial and sagittal arterial phase image with S.S.D. rendering

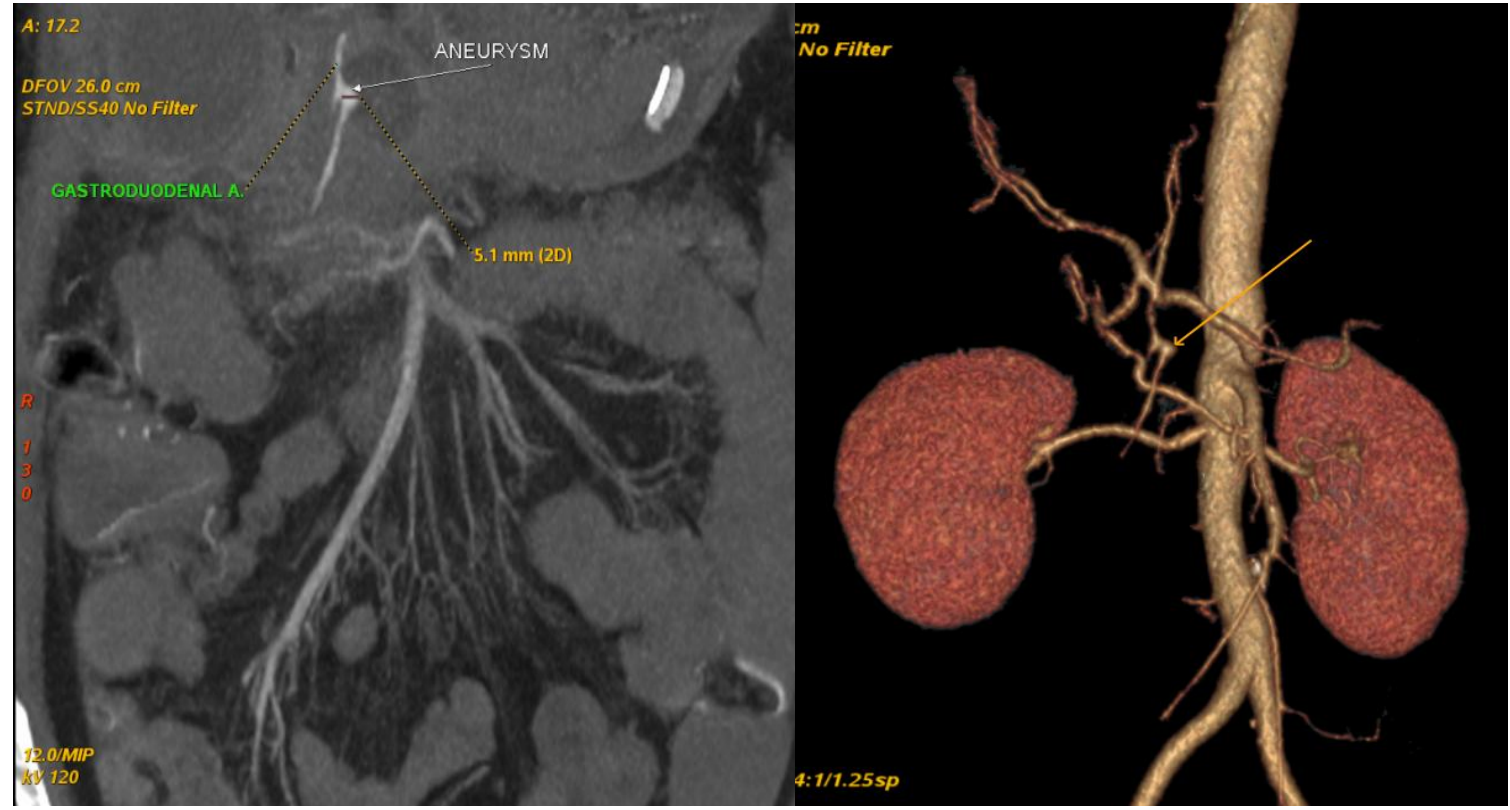

Figure $8(\mathbf{c}, \mathbf{d})$ post contrast coronal image with SSD rendered and VR rendered image

Figure 8 - A case of gastroduodenal artery aneurysm causing hematemesis

(viii) Varices

1(2.7\%) patient had multiple varices and had presented with hematemesis. He was a known case of liver cirrhosis and CT scan had shown multiple large esophageal, coronary venous, gastric, para-umbilical and spleno-renal varices, with evidence of portal hypertension and portal vein thrombosis (Fig.9). The most common cause of upper gastro-intestinal bleeding are peptic ulcer and oesophago-gastric varices.Coronary venous sinus varices were the most commonly found on CT scan $(80 \%)$ followed by esophageal varices $(45 \%){ }^{(15)}$. Risk of bleeding depends upon size of varices (if the caliber measures greater than $4 \mathrm{~mm}$ ) ${ }^{(16)}$ as well as presence of portal venous thrombosis ${ }^{(17)}$. 


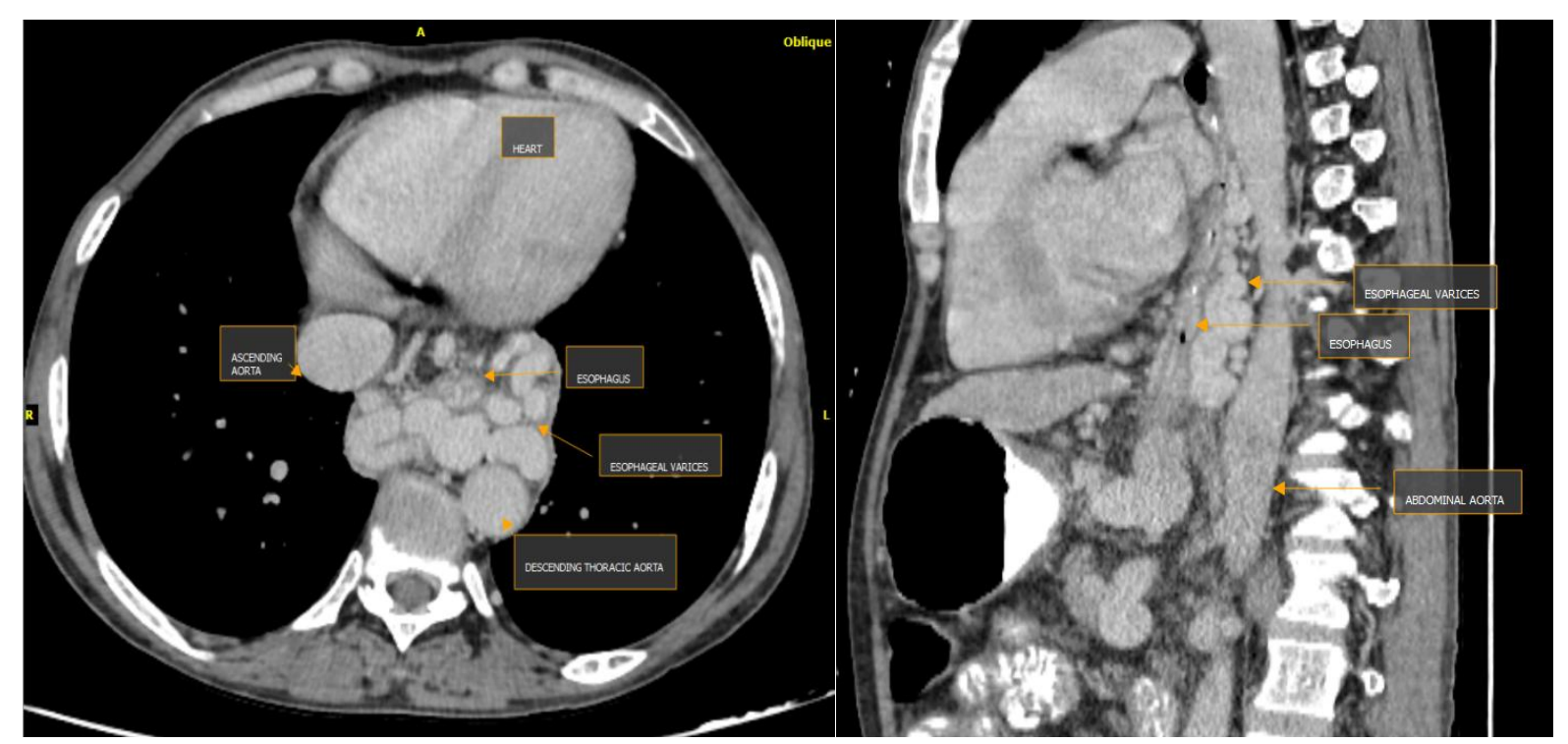

Fig.9 (a,b) contrast enhanced axial image and sagittal image (venous phase)

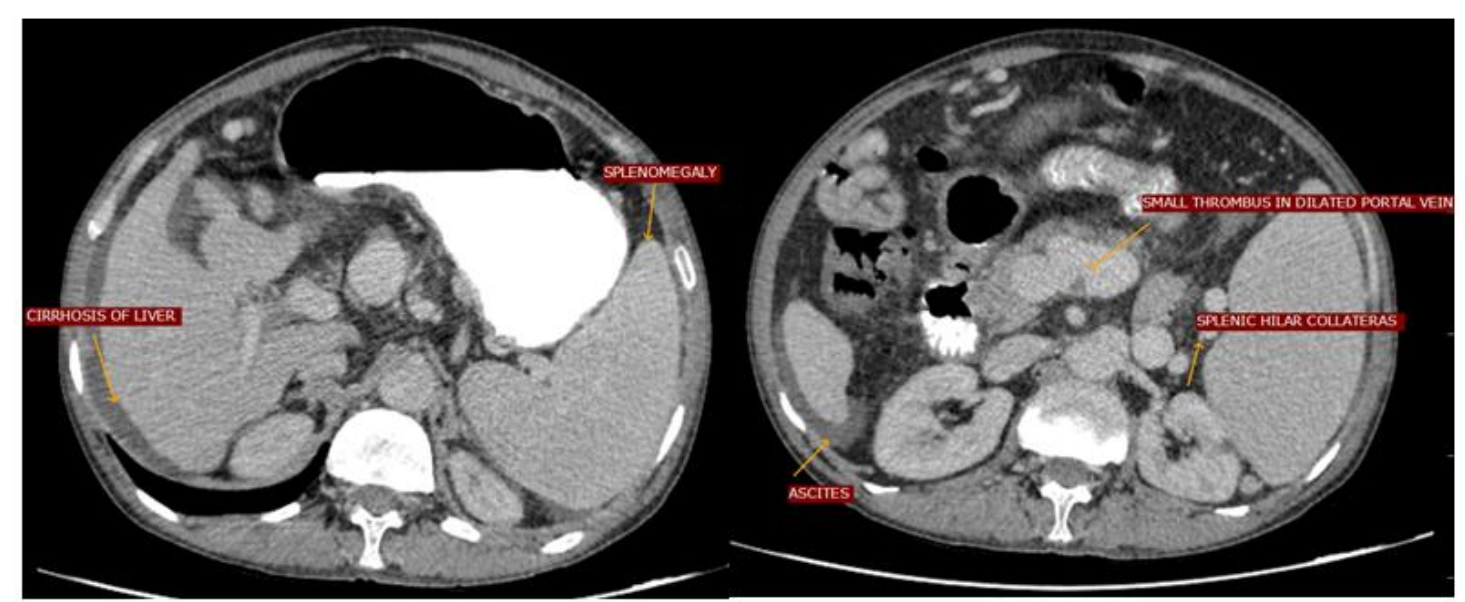

Fig. 7 (c) and (d) contrast enhanced axial images (venous phase)

Figure 9(a-d) - A case of esophageal and gastric varices causing hematemesis

\section{Conclusion}

Although CT scans are associated with exposure of patient by radiation and contrast related complications, these appear to be within maximum permissible limits, with great precautions taken to avoid any procedure induced or constrast related adversities. An adequately performed contrast MDCT examination; was thus found to be a promising first-line modality for the time-efficient, sensitive, and accurate diagnosis of the primary disease and determining the presence, location and severity of complications as a result of the disease. This had a profound impact on the subsequent treatment line of patients who presented with gastrointestinal bleeding.The findings of my study throw some light on the role of CT in gastrointestinal bleeding management in the setting of a developing country like ours and guide further research in the direction of cost effectiveness and optimization of use of MDCT in gastrointestinal bleeding diagnosis and management.

\section{References}

[1]. Joseph K. T., Stuart S. Sagel, Robert J. Stanley, and Jay P. Heiken. Computed Body Tomography with MRI Correlation. (EstadosUnidos: Lippincott Williams \& Wilkins, 2006).

[2]. Haaga, John R. CT and MRI of the Whole Body (Philadelphia, PA: Mosby/Elsevier, 2009)

[3]. Emil J. Balthazar, CT of the Gastrointestinal Tract: principles and interpretation American Journal of Roentgenology 156(1), 1991, 23-32.

[4]. S James, DM Balfe, JK Lee, and D Picus,Small-bowel disease: categorization by CT examination American Journal of Roentgenology, 148(5), 1987, 863-868.

[5]. James E. Huprich, Joel G. Fletcher, Jeffrey A. Alexander, Jeff L. Fidler, Sharon S. Burton, and Cynthia H. McCullough, Obscure Gastrointestinal Bleeding: Evaluation with 64-Section Multiphase CT Enterography-Initial Experience. Radiology246:2, 2008, $562-571$. 
[6]. Erik K. Insko, Marc S. Levine, Bernard A. Birnbaum, and Jill E. Jacobs. Benign and Malignant Lesions of the Stomach: Evaluation of CT Criteria for Differentiation Radiology, 228 (1), 2003, 166-171.

[7]. Serour, F., Dona, G., Birkenfeld, S., Balassiano, M. and Krispin, M. , Primary neoplasms of the small bowel. J. Surg. Oncol., 49(1), 1992,29-34.

[8]. Lewin, K. J., Ranchod, M. and Dorfman, R. F., Lymphomas of the gastrointestinal tract. A study of 117 cases presenting with gastrointestinal disease. Cancer, 42(2), 1978, 693-707.

[9]. Ruedi F. Thoeni and John P, Cello CT imaging of colitis. Radiology, 240:3, 2006, 623-638.

[10]. Fili KM, Abrams RA, Fishman EK, Spiral CT of colon cancer: Imaging features and role in management. Radiographics, 20(2), 2000, 419-430.

[11]. Laishram RS, Kaiho N, Shimray R, Devi SB, Punyabati P, Sharma DC. Histopathological evaluation of colorectal carcinomas status in Manipur, India. International Journal Of Pathology, 8(4), 2010, 5-8.

[12]. Sandra Beatriz Marion Valarini et al, Correlation between location, size and histologic type of colorectal polyps at the presence of dysplasia and adenocarcinoma, Journal of Coloproctology, 31(3), 241-247.

[13]. Khatibzadeh N, et al., Indirect role of site distribution in high-grade dysplasia in adenomatous polyps:J Cancer Res Ther, 4(1), 204207.

[14]. Ray G, Banerjee PK, Ghoshal UC et al, Etiology and management of obscure gastrointestinal bleed-an appraisal from eastern India. Indian J Gastroenterol20(3), 2001, 90-93.

[15]. K C Cho, Y D Patel, R H Wachsberg, and J Seeff, Varices in portal hypertension: evaluation with CT.RadioGraphics15(3), 1995, 609-622.

[16]. Young Jun Kim, Steven S. Raman, Nam C. Yu, Katherine J. To'o, Rome Jutabha, and David S. K. Lu, Esophageal Varices in Cirrhotic Patients: Evaluation with Liver CT; American Journal of Roentgenology 188(1), 2007, 139-144.

[17]. G. D'Amico and R. De Franchis, Upper digestive bleeding in cirrhosis. Post-therapeutic outcome and prognostic indicators Hepatology 38 (3), 2003, 599-612.

[18]. Liao, Zhuan et al, Indications and detection, completion, and retention rates of small-bowel capsule endoscopy: a systematic review Gastrointestinal Endoscopy , 71(2), 280 - 286.

[19]. Ríos A, Montoya MJ, Rodríguez JM, Serrano A, Molina J, Parrilla P, Acute lower gastrointestinal hemorrhages in geriatric patients. Dig Dis Sci 50(5), 2005, 898-904. 